\title{
Eventos tromboembólicos y el embarazo: actualización en anticoagulación en mujeres embarazadas
}

Thromboembolic events and pregnancy: an update on anticoagulation in pregnant women

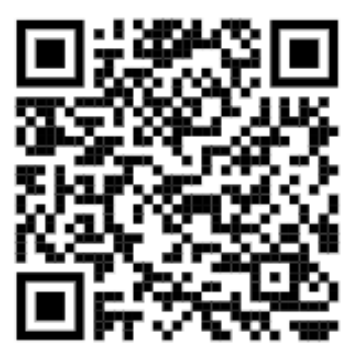

${ }^{1}$ Médico general, graduada de la Universidad de Costa Rica (UCR). Código médico: 15816 anaelena.cs25@gmail.com.

${ }^{2}$ Médico general, graduada de la Universidad de Costa Rica (UCR). Código médico: 15876 anamilems@gmail.com.

${ }^{3}$ Médico general, graduada de la Universidad de Costa Rica (UCR). Código médico: 15875 acamilamartin@gmail.com.

\author{
${ }^{1}$ Dra. Ana Elena Camacho Solís \\ Investigadora independiente, San José, Costa Rica \\ anaelena.cs25@gmail.com \\ https://orcid.org/0000-0001-5276-1432 \\ ${ }^{2}$ Dra. Ana Milena Méndez Zamora \\ Investigadora independiente, San José, Costa Rica \\ anamilems@gmail.com \\ https://orcid.org/0000-0003-0414-7336 \\ ${ }^{3}$ Dra. Ana Camila Martín Zamora \\ Investigadora independiente, San José, Costa Rica \\ acamilamartin@gmail.com \\ https://orcid.org/0000-0003-3004-3207
}

RECIBIDO

CORREGIDO

ACEPTADO

$30 / 03 / 2019$

$04 / 04 / 2019$

$16 / 04 / 2019$

\section{RESUMEN}

El embarazo se asocia a múltiples cambios fisiológicos y anatómicos que llevan a un estado protrombótico. Este estado incrementa 5 veces el riesgo de tromboembolismo venoso. Además, durante el periodo de gestación y puerperio existen otras condiciones, independientes o relacionadas con el embarazo, en las cuales la tromboprofilaxis farmacológica o la anticoagulación están indicadas. El iniciar la terapia anticoagulante en la mujer embarazada implica un balance de riesgos y beneficios para la madre y el feto así como consideraciones especiales según el tiempo de gestación. La heparina de bajo peso molecular es el tratamiento de elección en la mayoría de las indicaciones, sin embargo, existen casos en los que la warfarina o los anticoagulantes orales directos son de utilidad y están indicados como primera línea.

PALABRAS CLAVE: trombosis; tromboembolismo venoso; embarazo; anticoagulantes; heparina; warfarina. 


\begin{abstract}
Pregnancy is associated with multiple physiological and anatomical changes that make it a prothrombotic state. The combination of such factors results in a 5 -fold increase in the risk for venous thromboembolism in these patients. There are multiple conditions, independent or related to pregnancy, in which there is an indication for pharmacological thromboprophylaxis or anticoagulation during pregnancy and the postpartum period. In order to start anticoagulant therapy in a pregnant woman the risks and benefits for both the woman and the fetus should be taken into consideration according to the time of gestation. Even though low molecular weight heparins are the treatment of choice for most indications, there are cases in which warfarin or direct oral anticoagulants are of use and may be the first line of treatment.
\end{abstract}

KEYWORDS: thrombosis; venous thromboembolism; pregnancy; anticoagulants; heparin; warfarin.

\section{INTRODUCCIÓN}

La decisión de anticoagular a una mujer embarazada implica un delicado balance de los riesgos tromboembólicos que posee la madre y los riesgos de hemorragia del feto. Los compuestos de heparina, especialmente la heparina de bajo peso molecular (HBPM), por su seguridad en el embarazo han llegado a ser los fármacos de elección ante la mayoría de los escenarios clínicos, sin embargo, no siempre son la primera línea de tratamiento. Por esta razón, integrar las indicaciones de uso, la farmacocinética y la farmacodinamia de los anticoagulantes con la fisiología del embarazo para la respectiva edad gestacional es vital para poder individualizar los casos. De esta manera, se pretende lograr una selección idónea y juiciosa del anticoagulante a utilizar como primera línea con el objetivo de alcanzar un balance de riesgos capaz de llevar a un resultado óptimo tanto para la madre como para el feto. Este artículo pretende hacer una revisión de la literatura que le permita al clínico enfrentarse con seguridad ante la compleja decisión de anticoagular a una paciente embarazada $(1,2)$.

\section{PATOGÉNESIS DE LOS EVENTOS TROMBÓTICOS DURANTE EL EMBARAZO}

El embarazo se asocia a cambios anatómicos y fisiológicos que aumentan el riesgo de trombosis, al presentar alteraciones que contribuyen a los tres factores incluidos en la tríada fisiopatológica de Virchow. Dichos factores, que incluyen hipercoagulabilidad, estasis venosa y lesión endotelial, se presentan en distintos grados y momentos durante el transcurso del embarazo (3). La combinación de estos factores resulta en el incremento en 5 veces del riesgo de 
tromboembolismo venoso (TEV) en mujeres embarazadas, en comparación con la población general (4). Este riesgo aumenta conforme progresa el embarazo, estando presente desde el primer trimestre de gestación, inclusive antes de la aparición de los cambios anatómicos significativos. $Y$ a pesar de que el riesgo es mayor en el tercer trimestre, en comparación con el primer o segundo trimestre, el mayor riesgo se presenta durante el puerperio, especialmente durante la primera semana postparto (3). Lo anterior se ilustra por la observación de que la mitad de todos los eventos trombóticos asociados al embarazo ocurren en los 9 meses de gestación, pero que el restante $50 \%$ ocurre en las primeras 6 semanas después del parto. Después de 6 semanas el riesgo de trombosis se mantiene levemente elevado hasta los 3 meses postparto, para normalizarse posteriormente a los valores preconcepcionales (5).

La patogénesis de los eventos trombóticos asociados al embarazo es multifactorial y heterogénea, pero se destaca al estado de hipercoagulabilidad inducida por los cambios hormonales como uno de los principales factores asociados (4). Dicho estado está relacionado con el aumento fisiológico en la actividad de diversos factores procoagulantes con un descenso concomitante en la actividad de factores anticoagulantes, y con la inhibición de la fibrinólisis (5). En el embarazo hay un aumento en las concentraciones del fibrinógeno, el factor de von Willebrand y de los factores VII, VIII, X, los cuales tienden a incrementar la propensión a la formación de coágulos (6). Asimismo, se da un descenso del $40-60 \%$ en la actividad de la proteína $S$, resultado de la disminución en la producción total de proteína $\mathrm{S}$ inducida por estrógenos y de un aumento en la proteína ligadora de C4b, que se une a la proteína $S$ (7). Adicionalmente, hay una reducción en la fibrinólisis, asociado a un aumento de 5 veces en los niveles del inhibidor del activador del plasminógeno 1, y al aumento marcado durante el tecer trimestre del inhibidor del activador del plasminógeno 2 asociado a su producción placentaria (7).

Por otra parte, ciertos factores anatómicos asociados con el embarazo contribuyen a la estasis venosa. En las mujeres embarazadas, la presión ejercida por el útero grávido sobre la vasculatura pélvica y la vena cava inferior promueve un enlentecimiento del retorno venoso de las extremidades inferiores, lo cual aumenta conforme progresa la gestación (7). Cuando ocurre trombosis venosa profunda durante el embarazo, es más probable que sea de carácter proximal y que involucre la extremidad inferior izquierda, con frecuente asociación de las venas iliacas e iliofemoral. Esta distribución se asocia con el aumento de la estasis venosa en la pierna izquierda, por la compresión de la vena iliaca izquierda por parte de la arteria iliaca derecha, además de la compresión de la vena cava inferior por el útero grávido (3). Finalmente, la lesión endotelial, el último componente de la tríada de Virchow, ocurre durante el parto y la cesárea (7). Es importante destacar que la vía de parto también está relacionada con el riesgo de TEV. El parto por cesárea aumenta 4 veces el riesgo de TEV en comparación con el parto vaginal. Adicionalmente, el riesgo de TEV aumenta si se presentan complicaciones del parto en el puerperio, 
como la hemorragia postparto o infección (3).

Con respecto a los factores de riesgo asociados a TEV en mujeres gestantes, éstos pueden ser independientes 0 asociados con la gestación. El antecedente personal de trombosis es el factor de riesgo individual más importante para TEV en este periodo, ya que el riesgo de TEV recurrente aumenta de 3 a 4 veces en estas mujeres, y el 15 a $25 \%$ de todos los casos de TEV durante el embarazo son eventos recurrentes. El siguiente factor de riesgo más importante es la presencia de trombofilias, tanto hereditarias como adquiridas, al describirse su presencia en un 20 a 50\% de los casos (3). Otros factores de riesgo que contribuyen al riesgo de trombosis son los antecedentes heredofamiliares de eventos trombóticos, edad mayor a 35 años, obesidad, hipertensión arterial, diabetes, enfermedades inflamatorias crónicas, y condiciones asociadas con el embarazo, como gestaciones múltiples, preeclampsia, síndrome de HELLP e inmovilización (5).

\section{ANTICOAGULACIÓN EN EL EMBARAZO}

Existen múltiples condiciones en las que se requiere alcanzar la anticoagulación durante el embarazo y el postparto. Las mujeres que ya recibían tratamiento anticoagulante por alguna indicación como lo son historia de trombosis no provocada, trombosis recurrente, trombofilias, válvulas mecánicas o síndrome antifosfolípido (SAF), deben continuar con éste, ameritando según el caso una transición a un fármaco con conocida seguridad en el embarazo (1).
Es importante estratificar el riesgo individualmente en cada paciente para determinar el tipo de tratamiento que requerirá durante dicho periodo. Las mujeres con eventos agudos como TEV durante el embarazo también requerirán anticoagulación sistémica (6).

\section{Trombofilias y tromboembolismo venoso}

El Colegio Americano de Obstetricia y Ginecología (ACOG) da recomendaciones específicas para la anticoagulación en este tipo de pacientes según sus características y nivel de riesgo. Las mujeres sin trombofilia, con historia de un sólo episodio de TEV asociado a estrógenos o embarazo deben recibir anticoagulación en dosis profiláctica durante todo el embarazo y 6 semanas postparto, debido al riesgo de potencial recurrencia. Las mujeres con historia de un episodio de TEV no provocado por un motivo conocido y aquellas con historia de 2 o más episodios de TEV se consideran de alto riesgo, y por lo tanto deben también ser manejadas con anticoagulación, con una dosis más elevada en el postparto. Por otro lado, aquellas con historia de un único episodio provocado por un motivo conocido como cirugía, trauma o inmovilización (sin relación a estrógenos o embarazo) no requerirán tratamiento, se recomienda únicamente observación $(3,9)$.

En el caso de pacientes portadoras de una trombofilia considerada de bajo riesgo como heterocigotas del factor $\mathrm{V}$ de Leiden, heterocigotas de la mutación del gen de protrombina y portadoras de una deficiencia de proteína $C \circ S$, se manejarán según sus características e historia personal. Aquellas sin historia 
personal de TEV y sin historia familiar de importancia no requerirán anticoagulación. Si por otro lado, poseen un factor de riesgo adicional como historia familiar de TEV en un familiar de primer grado, deberán recibir anticoagulación en dosis profilácticas únicamente en el periodo de 6 semanas postparto. Mientras tanto, el poseer historia personal de un episodio de TEV las hará candidatas a recibir anticoagulación en dosis profilácticas durante todo el periodo de gestación y postparto (3). Por otro lado, las mujeres con trombofilias de alto riesgo como homocigotas del factor $\mathrm{V}$ de Leiden, homocigotas de la mutación del gen de protrombina, heterocigotas con ambas mutaciones $\mathrm{o}$ aquellas portadoras de deficiencia de antitrombina, deberán ser todas tratadas con anticoagulación en dosis profilácticas (3).

\section{Válvulas mecánicas}

Este apartado se explica a profundidad en el apartado de la warfarina.

\section{Síndrome antifosfolípido (SAF)}

El SAF se caracteriza por un riesgo aumentado ambos de eventos trombóticos y morbilidad en el embarazo, por lo que es de vital importancia tratarlo en la población de mujeres gestantes. En mujeres embarazadas con SAF e historia de pérdidas gestacionales recurrentes (3 o más), estudios han demostrado que agregar heparinas de bajo peso molecular a la aspirina que asocian a un menor riesgo de pérdida que al utilizar aspirina únicamente $(10,11)$. Por lo tanto, se recomienda el uso de ambos fármacos en dichas pacientes $(6,11)$. Aquellas mujeres portadoras de SAF con historia de eventos trombóticos, siempre necesitarán de anticoagulación durante el embarazo. Las pacientes con historia de eventos trombóticos sin anticoagulación preembarazo, deberán utilizar HBPM a dosis profilácticas durante la gestación y postparto. Aquellas que por el contrario sí utilizan anticoagulación fuera del embarazo, deberán utilizar HBPM a dosis terapéuticas durante el mismo periodo (6).

\section{Tromboembolismo venoso agudo}

En mujeres embarazadas que presenten un episodio de TEV agudo, se deberá iniciar tratamiento inmediatamente ya sea con HNF o HBPM a dosis terapéuticas. Debido a que no se conoce el tiempo óptimo para el tratamiento de estas pacientes, basándose en los datos de la población no gestante, se recomienda un mínimo de 3 meses de tratamiento anticoagulante posterior al evento. Como el riesgo de trombosis continúa durante el embarazo y 6 semanas postparto, se recomienda continuar el tratamiento durante todo este tiempo aunque el evento haya sucedido al inicio del embarazo (6).

\section{FÁRMACOS ANTICOAGULANTES EN EL EMBARAZO}

\section{- Heparina}

Actualmente, la heparina no fraccionada (HNF) y la HBPM son los anticoagulantes más utilizados dentro de la categoría de anticoagulantes parenterales. Éstos son parte de los anticoagulantes parenterales indirectos, ya que su actividad está mediada por cofactores plasmáticos (12). El efecto anticoagulante de la heparina se descubrió inicialmente en el 1916, pero no fue hasta los años 1930 que se desarrolló la HNF como compuesto (13). El mecanismo de acción de la HNF se basa en aumentar la actividad de la antitrombina en la inactivación de tanto la trombina como el factor $\mathrm{Xa}$, mediante un 
cambio en su conformación (12). Su vía de administración es por medio de inyección subcutánea 0 infusión intravenosa, pero presenta el inconveniente que su farmacocinética no es predecible. Por lo anterior, cuando se utilizada para anticoagulación, se requiere monitoreo de su actividad y ajustes según el tiempo parcial de tromboplastina (TPT) (13).

Posteriormente, en los años 1980, se desarrolló la HBPM, por medio de la despolimerización de las cadenas de HNF (13). La HBPM también actúa al favorecer la actividad de la antitrombina, pero presenta un mayor efecto en la inhibición del factor Xa que de la trombina (12). Su vía de administración es por inyección subcutánea, tanto para su uso como tromboprofilaxis o anticoagulación. Con respecto a sus características farmacocinéticas, la HBPM presenta una farmacocinética predecible y una vida media mayor (aproximadamente 4 horas), lo que permite establecer esquemas de dosificación específicos para cada indicación según el peso materno, sin la necesidad de monitorización estricta (13). Algunos estudios pequeños sugieren la posibilidad de utilizar los niveles de antifactor Xa para el ajuste de la dosis de HBPM, pero se argumenta que las mujeres embarazadas pueden no requerir dicha monitorización al no haber estándares establecidos en esta población específica del valor óptimo de éste 0 de la frecuencia de su monitorización (8).

Durante décadas se han utilizado las heparinas en el contexto de mujeres embarazadas. Tanto la HNF como la HBPM no son capaces de cruzar la barrera placentaria y no se han asociado con efectos teratogénicos, por lo que son consideradas la primera línea de terapia farmacológica en la mayoría de las indicaciones de anticoagulación en mujeres embarazadas. Adicionalmente, su absorción por vía oral no es significativa, por lo que su uso durante la lactancia es seguro a pesar de estar presentes en cantidades mínimas en la leche materna (6). Por su perfil de seguridad superior y la facilidad de su administración, el Colegio Americano de Obstetricia y Ginecología, ACOG, recomienda el uso de la HBPM sobre la HNF para la prevención y tratamiento de TEV en mujeres embarazadas. A pesar de que hay pocos estudios comparativos del uso de HBPM en el embarazo, múltiples estudios en la población no embarazada han asociado a la HBPM con menos resultados adversos en comparación con la HFN. Algunas posibles ventajas a corto y largo plazo del uso de la HBPM incluyen menos episodios de sangrado, una respuesta terapéutica más predecible, menor riesgo de trombocitopenia inducida por heparina, y menos pérdida de densidad mineral ósea (3). En la TABLA 1 se detallan los esquemas de dosificación de heparina más comúnmente utilizados durante el embarazo, tanto en mujeres embarazadas con indicación para profilaxis como para anticoagulación (8). Las mujeres con indicación para anticoagulación idealmente deben recibir consejería preconcepcional.

En las mujeres en terapia con anticoagulantes orales directos, éstos se deben sustituir por warfarina o HBPM cuando inician los intentos por concebir, ya que no hay evidencia de los posibles 


\begin{tabular}{|c|c|}
\hline Dosis profiláctica & Dosis terapéutica \\
\hline \multicolumn{2}{|l|}{ Heparina de bajo peso molecular } \\
\hline $\begin{array}{l}\text {-Enoxaparina, } 40 \text { mg subcutáeneo } 1 \text { vez por día } \\
\text {-Dalteparina, } 5000 \text { unidades subcutáneo } 1 \text { vez por } \\
\text { día }\end{array}$ & $\begin{array}{l}\text {-Enoxaparina } 1.5 \mathrm{mg} / \mathrm{kg} 1 \mathrm{vez} \text { al día o } 1 \mathrm{mg} / \mathrm{kg} \\
\text { cada } 12 \text { horas subcutáneo } \\
\text {-Dalteparina } 200 \text { unidades } / \mathrm{kg} 1 \mathrm{vez} \text { al día o } \\
100 \text { unidades } / \mathrm{kg} \text { cada } 12 \text { horas }\end{array}$ \\
\hline \multicolumn{2}{|l|}{ Heparina no fraccionada } \\
\hline $\begin{array}{l}\text {-HNF } 5000-7500 \text { unidades subcutáneo cada } 12 \\
\text { horas durante el primer trimestre } \\
\text {-HNF } 7500-10000 \text { unidades subcutáneo cada } 12 \\
\text { horas durante el segundo trimestre } \\
\text {-HNF } 10000 \text { unidades subcutáneo cada } 12 \text { horas } \\
\text { durante el tercer trimestre }\end{array}$ & $\begin{array}{l}\text {-HNF en una dosis inicial de } 250 \\
\text { unidades } / \mathrm{kg}^{*} \text { subcutáneo cada } 12 \text { horas, } \\
\text { ajustado posteriormente para alcanzar un } \\
\text { TPT dentro del rango terapéutico } 6 \text { horas } \\
\text { después de la inyección. }\end{array}$ \\
\hline
\end{tabular}

efectos de la exposición en el embarazo temprano. Si las mujeres con deseos de concebir se encuentran utilizando warfarina, se recomienda el uso de pruebas de embarazo desde el primer día de atraso en el ciclo menstrual, y de ser éste resultado positivo, se debe sustituir éste fármaco por HBPM y revertir los efectos de la warfarina mediante suplementos orales de vitamina K. Se debe realizar este cambio antes de las 6 semanas de gestación, por los posibles efectos teratogénicos asociados a los antagonistas de la vitamina $\mathrm{K}(6)$.

En el contexto de TEV de diagnóstico durante el embarazo, la HBPM es la primera línea de tratamiento. Una revisión de Cochrane de 22 estudios de pacientes con TEV demostró que la HBPM fue superior a la HNF, al asociar menor tasa de recurrencia de TEV o extensión de ésta, menores episodios de sangrado mayor después de iniciado el tratamiento y menor mortalidad total. En estas pacientes se utilizan los esquemas terapéuticos ajustados según peso, y por vía subcutánea. (LIM) El uso de HNF intravenoso puede ser considerado como el tratamiento inicial en casos de tromboembolismo pulmonar 0 en situaciones en las cuales pueda ser necesario la inducción del parto, cirugía o trombolisis (3).

Las decisiones acerca del momento del parto en pacientes en tratamiento con HBPM O HNF se deben basar en las indicaciones obstétricas usuales, tomando en cuenta como meta el mantener una anticoagulación adecuada durante el embarazo al igual que evitar un efecto adverso durante el parto que esté asociado a la coagulación (3). Se recomienda que en las mujeres que reciben HBPM como profilaxis se descontinúe el fármaco al menos 12 horas antes de la inducción del parto o parto por cesárea. De igual forma, se debe descontinuar la HBPM al menos 24 horas antes en las mujeres que reciben niveles terapéuticos para anticoagulación. En ambos casos, se debe esperar dicho intervalo de tiempo establecido antes del 
uso de anestesia neuroaxial, por el riesgo de hematoma epidural o espinal. En los casos de inicio espontáneo de la labor de parto, se debe suspender el uso de HBPM en el momento que inician las contracciones 0 si hay ruptura de membranas (5). Por otra parte, las mujeres que reciben dosis de HNF mayores a 7500 unidades subcutáneas dos veces al día, se recomienda suspender el fármaco al menos 12 horas antes, además de la evaluación por laboratorio de los parámetros de la coagulación (3).

Con respecto a la reiniciación de la terapia anticoagulante en el postparto, se recomienda no reiniciarla antes de transcurridas 4 a 6 horas posterior a un parto vaginal, o 6 a 12 horas posterior a parto por cesárea. Además, en los casos de bloqueo neuroaxial, se recomienda diferir el reinicio de HBPM por al menos 24 horas después de su aplicación y 4 horas posterior a la suspensión del catéter. Posteriormente, en las mujeres que requieren menos de 6 semanas de terapia posterior al parto, usualmente se utiliza la HPBM como tratamiento, ya que la utilidad de la warfarina es limitada porque usualmente se requieren de 1 a 2 semanas para alcanzar los rangos terapéuticos (3).

\section{- Warfarina}

La warfarina es el anticoagulante oral más antiguo que existe. Su descubrimiento remonta a 1939 donde fue identificado, por primera vez, el dicumarol. A partir de este compuesto se elaboró un congénere sintético al que se le llamó warfarina. Este fármaco es un antagonista de la vitamina K. Su mecanismo de acción es a través de la inhibición de la enzima vitamina $K$ epóxido reductasa. Al inhibir dicha enzima, no se logra regenerar la vitamina $K$ reducida que es necesaria para la carboxilación que permite la síntesis de proteínas biológicamente activas. Por lo tanto, los factores dependientes de vitamina $\mathrm{K}$ permanecerán como prozimógenos no funcionales dándole a la warfarina su efecto anticoagulante (14).

El uso de la warfarina en el embarazo se viene estudiando desde hace más de cuatro décadas. Se contraindicó por primera vez a finales de 1970, pero hasta 1980 se publicó el primer estudio que reportaba los efectos adversos de dicho fármaco en el embarazo. En el 2002 se publicó un segundo estudio que confirmaba dichos efectos adversos. Gracias a estas dos publicaciones se sabe que el uso de la warfarina en embarazadas puede originar defectos congénitos y aborto, sin embargo sí existen indicaciones para su uso durante la gestación (15). Los efectos adversos de la warfarina se dan gracias a que cruza libremente la barrera placentaria, pero estos varían de acuerdo con el trimestre del embarazo en el cual se esté utilizando. Durante el primer trimestre, está asociada a un síndrome fetal caracterizado por hipoplasia nasal y calcificaciones epifisiarias puntiformes similares a la condrodisplasia punteada. La teoría fisiopatológica que sustenta dicho síndrome fetal es que la inhibición de la vitamina $K$ epóxido reductasa inhibe la formación de vitamina $K$ reducida que es la encargada de carboxilar dos componentes esenciales para el depósito de calcio $y$, por ende, la formación de hueso. Este proceso de osificación ósea tiene su periodo crítico de las 6 a las 12 semanas de gestación. Por lo tanto, la interrupción de su uso antes de las 6 
semanas puede prevenir la embriopatía warfarínica. Durante el segundo o tercer trimestre del embarazo, el uso de warfarina se ha asociado a mayor riesgo de muerte fetal intra útero, aborto, muerte neonatal y, principalmente, a riesgo de sangrado. El riesgo de sangrado es especialmente importante a nivel de sistema nervioso central ya que puede llevar a anormalidades del mismo como retardo mentral, ceguera, espasticidad y convulsiones en el niño. Este riesgo aumenta para el feto aún más durante el parto vaginal por lo que alguna literatura recomienda realizar una cesárea una vez iniciada la labor de parto en mujeres anticoaguladas con warfarina $(1,2,15)$.

La anticoagulación con warfarina durante el embarazo ha sido ampliamente sustituida por la anticoagulación con los compuestos de heparina, especialmente la heparina de bajo peso molecular (HBPM). La única excepción es en mujeres embarazadas con una válvula cardiaca mecánica. En este caso, múltiples estudios han demostrado una mayor incidencia de eventos trombóticos asociados al uso de compuestos de heparina. Uno de estos estudios demostró una incidencia de $3.9 \%$ de eventos trombóticos en mujeres embarazadas tomando warfarina a lo largo del embarazo versus $9.2 \%$ si recibían heparina no fraccionada (HNF) en el primer trimestre seguido de warfarina en el segundo y tercer trimestre o de $33 \%$ si recibían HNF durante todo el embarazo. Además, aunque todavía en discusión, se cree que los efectos adversos de dicho fármaco son dosis dependiente y se presentan con menor incidencia en mujeres que toman menos de $5 \mathrm{mg}$ cada día de warfarina (16). Las mujeres con válvulas cardiacas mecánicas presentan, por las razones expuestas, un conflicto para el clínico de riesgo beneficio para la madre y el feto a la hora de seleccionar un anticoagulante. Ante dicha polémica, el Colegio Americano de Cardiología y la Asociación Americana de Corazón (ACC/AHA, por sus siglas en inglés) dieron recomendaciones para estos casos específicos en sus guías de anticoagulación. Ellos brindan cuatro recomendaciones con nivel de evidencia I. La primera es que las mujeres con válvulas cardiacas mecánicas anticoaguladas con warfarina deben tener un INR terapéutico de 2-3 (INR de 2,5- 3,5 para válvulas mecánicas mitrales). En la segunda dicen que, en mujeres embarazadas, se puede utilizar warfarina para llegar a niveles terapéuticos de INR durante el segundo y tercer trimestre del embarazo. De tercero recomiendan que se debe descontinuar la warfarina e iniciar HNF intravenosa antes del parto vaginal con un objetivo de TPT dos veces mayor que el valor que se tomó de control. Por último, su cuarta recomendación es agregar aspirina a dosis bajas (75 a 100 $\mathrm{mg}$ ) durante el segundo y tercer trimestre del embarazo (16).

Esta guía deja pautas más claras para el segundo y tercer trimestre con un buen nivel de evidencia. En el primer trimestre, dan dos recomendaciones con un nivel de evidencia Ila. La primera es continuar warfarina durante el primer trimestre del embarazo si la dosis para alcanzar un INR terapéutico es igual o menor a $5 \mathrm{mg}$ cada día y previo a discutir los riesgos con la paciente. La segunda recomendación es utilizar HBPM dos veces al día con un niveles anti-Xa de $0,8 \mathrm{IU} / \mathrm{mL}$ a 1,2 IU/mL medidos 4 a 6 horas después de la administración durante el primer trimestre del embarazo si la dosis diaria de 
warfarina supera los $5 \mathrm{mg}$ por día para alcanzar un INR terapéutico (17). En resumen, las guías proponen que la warfarina a bajas dosis (menores o iguales a $5 \mathrm{mg}$ cada día) están indicadas a lo largo de todo el embarazo en mujeres con válvulas cardiacas mecánicas. Este anticoagulante se debe sustituir por HBPM únicamente si se exceden los $5 \mathrm{mg}$ diarios y su cambio sería exclusivo para el primer trimestre. Cabe destacar que todas las recomendaciones brindadas exigen un estricto control prenatal desde el inicio del embarazo (1).El uso de warfarina durante el postparto es seguro ya que no se documentan concentraciones de dicho fármaco en la leche materna. Esto la convierte en una buena opción de anticoagulante oral en el postparto, sin limitaciones particulares como sí las que tiene durante el embarazo. Igual que en mujeres no embarazadas, se recomienda hacer una transición a warfarina con puente de HNF o HBPM. En pacientes con una válvula cardiaca mecánica se puede reiniciar la warfarina 24 horas después del parto con la debido transición usando compuestos de heparina como puente (3).

\section{Anticoagulantes orales directos}

La Sociedad Internacional de Trombosis y Hemostasia (ISTH) indica que los anticoagulantes orales directos - como dabigatrán, rivaroxabán, apixabán y edoxaban - no deben ser utilizados en mujeres embarazadas, planeando un embarazo o dando lactancia materna. En el caso de una mujer que quede embarazada de manera no intencional utilizándolos, recomiendan suspenderlos de inmediato y sustituirlos por HBPM (18). Lo anterior se debe a que se conoce que dichos medicamentos cruzan la placenta, y existe aún falta de evidencia confiable sobre sus efectos potenciales en feto (6). Cabe destacar que en un estudio realizado en una pequeña población de pacientes con exposición involuntaria a anticoagulantes orales directos en el embarazo, el riesgo de embriopatía resultó ser al menos no superior al de aquel descrito con antagonistas de la vitamina K (19).

\section{Fondaparinux}

El fondaparinux es un pentasacárido sintético inhibidor del factor $\mathrm{Xa}$, cuyo perfil de seguridad en el embarazo aún permanece incierto. Se conoce que dicho fármaco cruza la placenta, sin embargo no existen datos claros en cuanto a su relación con posibles malformaciones congénitas (6). Una publicación reciente documentó que en mujeres embarazadas utilizando el medicamento, la tasa de complicaciones en el embarazo fue similar a aquella de la población general (20). El Colegio Americano de Obstetricia y Ginecología (ACOG), recomienda Fondaparinux para anticoagulación en aquellas mujeres embarazados que hayan presentado ya sea alergias cutáneas severas con el uso de heparinas de bajo peso molecular o trombocitopenia inducida por heparina (TIH) (3). Debido a su larga vida media (17 horas), se recomiendan 5 días de tiempo entre su última dosis y la anestesia epidural (8).

\section{Danaparoid}

El danaparoid es un inhibidor indirecto del factor $\mathrm{Xa}$, actualmente considerado un fármaco de categoría B en el embarazo. Estudios han demostrado que no cruza la placenta y no se detecta en leche materna $(6,8)$. Se considera otra opción para la anticoagulación en embarazadas con 
historia de $\mathrm{TIH}$, no obstante, su uso es menos común debido a su disponibilidad limitada en varios países (21).

\section{Argobatrán}

El argobatrán es un inhibidor directo de la trombina (lla) intravenoso, por lo que su uso en el embarazo es más limitado. Es considerado categoría B en embarazo, aunque su evidencia en cuanto a seguridad en humanos proviene principalmente de un pequeño número de reportes de casos (8). Debido a su vida media corta de 40 minutos, permite un tiempo más corto entre su dosis y el uso de anestesia neuroaxial. Por lo tanto, un posible uso es en el momento de parto en una embarazada siendo tratada por $\mathrm{TIH}$ (6).

\section{CONCLUSIÓN}

La afectación de la triada de Virchow durante el embarazo lleva a que el mismo se caracterice por ser un estado protrombótico. Existen múltiples indicaciones para la anticoagulación durante el embarazo como lo son las trombofilias e historia de TEV, las válvulas cardiacas mecánicas, SAF, TEV agudo, entre otros. La HBPM sigue siendo la terapia de elección para la mayoría de las indicaciones de anticoagulación durante la gestación, por su perfil de seguridad al no cruzar placenta, sin embargo, su uso es diferido en ciertos casos. Este fármaco presenta múltiples ventajas con respecto a otros agentes anticoagulantes, pero es importante que su uso influye en las decisiones con respecto al momento del parto y a la aplicación de anestesia neuroaxial.

Con respecto a otros fármacos, la warfarina está indicada durante todo el embarazo en pacientes con válvulas cardiacas mecánicas si la dosis diaria para alcanzar un INR terapéutico es menor a $5 \mathrm{mg}$. Si la dosis diaria es mayor a ésta, se recomienda sustituir únicamente durante el primer trimestre con HPBM. Por otra parte, en los escenarios clínicos en los que se presente $\mathrm{TIH}$, se recomienda un cambio de anticoagulación a Fondaparinux, Danaparoid o Argobatrán. Estos han demostrado no tener un riesgo de embriopatía superior al de la warfarina, sin embargo, aún cuentan con pocos estudios que respalden su seguridad en esta población.

\section{REFERENCIAS}

1. Ishawabkeh L, Econocmy KE, Valente AM. Anticoagulation During Pregnancy: Evolving Strategies With a Focus on Mechanical Valves. J Am Coll Cardiol 2016; 68(16): 1804-1813. https://doi.org/10.1016/j.jacc.2016.06.076

2. Gibson PS, Powrie R. Anticoagulants and pregnancy: When are they safe? Cleve Clin J Med 2009; 76(2): 113-27. https://doi.org/10.3949/ccim.75a.072272

3. American College of Obstetricians and Gynecologists. Thromboembolism in pregnancy. ACOG Practice Bulletin No. 196. American College of Obstetricians and Gynecologists. Obstet Gynecol 2018; 132: e1-e17. https://doi.org/10.1097/AOG.0000000000002706

4. Sucker C, Zotz R. Prophylaxis and treatment of venous thrombosis and pulmonary embolism in pregnancy. Reviews in Vascular Medicine. 2015; 3(2): 24-30. https://doi.org/10.1016/i.rvm.2015.05.003

5. Scheres LJJ, Bistervels IM, Middeldorp S. Everything the clinician needs to know about evidencebased anticoagulation in pregnancy. Blood Rev. 2019; 33: 82-97 https://doi.org/10.1016/j.blre.2018.08.001 
6. Scheres LJJ, Bistervels IM, Middeldorp S. Everything the clinician needs to know about evidencebased anticoagulation in pregnancy. Blood Rev. 2019; 33:82-97. https://doi.org/10.1016/i.blre.2018.08.00

7. Lim A, Samarage A, Lim B. Venous thromboembolism in pregnancy. Obstetrics, Gynaecology \& Reproductive Medicine. 2016; 26(5): 133-139. https://doi.org/10.1016/i.ogrm.2016.02.005

8. Fogerty AE. Challenges of Anticoagulation Therapy in Pregnancy. Curr Treat Options Cardio Med. 2017; 19(10): 1-13. https://doi.org/10.1007/s11936-017-0575-x

9. Yarrington CD, Valente AM, Economy KE. Cardiovascular management in pregnancy: antithrombotic agents and antiplatelet agents. Circulation. 2015; 132: 1354-1364. https://doi.org/10.1161/CIRCULATIONAHA.114.003902

10. Ziakas PD, Pavlou M, Voulgarelis M. Heparin treatment in antiphospholipid syndrome with recurrent pregnancy loss: a systematic review and meta-analysis. Obstet Gynecol. 2010; 115(6): 1256-1262. https://doi.org/10.1097/AOG.0b013e3181deba40

11. Antovic A, Sennstrom M, Bremme K, Svenungsson E. Obstetric antiphospholipid syndrome. Lupus Sci Med. 2018; 5(1): e000197. https://doi.org/10.1136/lupus-2016-000197

12. Ferreira J, Wipf J. Pharmacologic Therapies in Anticoagulation. Medical Clinics of North America. 2016; 100(4): 695-718. https://doi.org/10.1016/i.mcna.2016.03.007

13. Breen K. Anticoagulation treatment. Medicine. 2017; 45(4): 239-243. https://doi.org/10.1016/i.mpmed.2017.01.010

14. Hogg K, Weitz J. Goodman y Gilman: The Pharmacological Basis of Therapeutics. 13th edition. Blood coagulation and Anticoagulant, Fibrinolytic, and Antiplatlet Drugs. New York.: McGraw-Hill; c2018: 592-593 p.

15. Blickstein D, Blickstein I. The risk of fetal loss associated with Warfarin anticoagulation. Int J Gynaecol Obstet 2002; 78(3): 221-225. https://doi.org/10.1016/S0020-7292(02)00186-8

16. Richardson A, Shah S, Harris C, McCulloch G, Antoun P. Anticoagulation for the Pregnant Patient with Mechanical Heart Valve, No Perfect Therapy: Review of Guidelines for Anticoagulation in the Pregnant Patient. Case Rep Cardiol. 2017; 2017: ID3090273. https://doi.org/10.1155/2017/3090273

17. Nishimura RA, Otto CM, Bonow RO, Carabello BA, Erwin JP 3rd, Fleisher LA, et al. 2017 AHA/ACC Focused UPdate of the 2014 AHA/ACC Guideline for the Management of Patients With Valvular Heart Disease: A Report of the American College of Cardiology/American Heart Association Task Force on Clinlcal Practice Guidelines. Circulation. 2017; 135(25): e1159-e1195. https://doi.org/10.1161/CIR.0000000000000503

18. Cohen H, Arachchillage DR, Middeldorp S, Beyer-Westendorf J, Abdul-Kadir R. Management of direct oral anticoagulants in women of childbearing potential: guidance from the SSC of the ISTH. J Thromb Haemost. 2016; 14(8): 1673-1676. https://doi.org/10.1111/ith.13366

19. Beyer-Westendorf J, Michalski F, Tittl L, Middeldorp S, Cohen H, Abdul Kadir R, et al. Pregnancy outcome in patients exposed to direct oral anticoagulants-and the challenge of event reporting. Thromb Haemost. 2016; 116(4): 651-658. https://doi.org/10.1160/TH16-04-0305

20. DeCarolis S, di Pasquo RE, Del Sordo G, Buonomo A, Schiavino D, Lanzone A, et al. Fondaparinux in pregnancy: could it be a safe option? A review of the literature. Thromb Res. 2015; 135(6): 10491051. https://doi.org/10.1016/i.thromres.2015.04.001

21. Mauermann $\mathrm{E}$ et al. Heparin-induced thrombocytopenia in pregnancy: an interdisciplinary challengea case report and literature review. Int $J$ Obstet Anesth. 2016; 26: 79-82. https://doi.org/10.1016/j.ijoa.2015.11.009 